\title{
AN INCONSISTENCY CONTROL SYSTEM BASED ON INCOMPLETE PAIRWISE COMPARISON MATRICES
}

\author{
Sándor Bozóki \\ Computer and Automation Research Institute \\ Hungarian Academy of Sciences (MTA SZTAKI); \\ Corvinus University of Budapest (BCE) \\ Budapest, Hungary \\ E-mail: bozoki@sztaki.hu; sandor.bozoki@uni-corvinus.hu
}

\begin{abstract}
Incomplete pairwise comparison matrix was introduced by Harker in 1987 for the case in which the decision maker does not fill in the whole matrix completely due to, e.g., time limitations. However, incomplete matrices occur in a natural way even if the decision maker provides a completely filled in matrix in the end. In each step of the total $n(n-1) / 2$, an incomplete pairwise comparison is given, except for the last one where the matrix turns into complete. Recent results on incomplete matrices make it possible to estimate inconsistency indices $C R$ and $C M$ by the computation of tight lower bounds in each step of the filling in process. Additional information on ordinal inconsistency is also provided. Results can be applied in any decision support system based on pairwise comparison matrices. The decision maker gets an immediate feedback in case of mistypes, possibly causing a high level of incons istency.
\end{abstract}

Keywords: Inconsistency, incomplete pairwise comparison matrix, multi-attribute decision models.

\section{Introduction}

In multi-attribute decision models, weighting the criteria and evaluating the alternatives with respect to the criteria are of the most important steps. In the paper, the method of pairwise comparison matrices [Saaty, 1980] and its generalization for the incomplete case is discussed. For simplic ity, assume that the objects to compare are the importances of $n$ criteria (the method is the same when comparing alternatives to each other, voting powers of the individuals in group decision making, or estimated probabilities of future events). A (complete) pairwise comparison matrix $\mathbf{A}=\left[a_{i j}\right]_{i, j}=1, \ldots, n$ is a matrix of size $n \times n$ with the properties as follows:

$$
\begin{aligned}
& a_{i j}>0 ; \\
& a_{i i}=1 ; \\
& a_{i j}=1 / a_{j i}
\end{aligned}
$$

for $i, j=1, \ldots, n$, where $a_{i j}$ is the numerical answer given by the decision maker for the question

- "How many times Criterion $i$ is more important than Criterion $j$ ?" or

- "How many times Alternative $i$ is better than Alternative $j$ with respect to a given criterion?" or

- "How many times Decision Maker $i$ has larger voting power than Decision Maker $j$ ? " in a group decision making problem.

- "How many times Event $i$ is more likely than Event $j$ ?" in a probability estimation problem. 
The weighting problem is to find the $n$-dimensional positive weight vector $\mathbf{w}=\left(w_{1}, w_{2}, \ldots, w_{n}\right)^{T}$ such that the appropriate ratios of the components of $\mathbf{w}$ reflect, or, at least, approximate all the $a_{i j}$ values $(i, j=1, \ldots, n)$, given by the decision maker. In the Analytic Hierarchy Process [Saaty, 1980] Eigenvector Method is applied and the approximation $\mathbf{w}^{E M}$ of $\mathbf{w}$ is defined by

$$
\mathbf{A} \mathbf{w}^{E M}=\lambda_{\max } \mathbf{w}^{E M},
$$

where $\lambda_{\max }$ denotes the maximal eigenvalue, also known as Perron eigenvalue, of $\mathbf{A}$ and $\mathbf{w}^{E M}=\left(w_{1}^{E M}, w_{2}^{E M}, \ldots, w_{n}{ }^{E M}\right)^{T}$ denotes the right-hand side eigenvector of $\mathbf{A}$ corresponding to $\lambda_{\max }$. By Perron's theorem, $\mathbf{w}^{E M}$ is positive and unique up to a scalar multiplication. The most often used normalization is $\Sigma w_{i}^{E M}=1$.

A pairwise comparison matrix in is called consistent if the transitivity

$$
a_{i j} a_{j k}=a_{i k}
$$

holds for all indices $i, j, k=1, \ldots, n$. Otherwise, the matrix is inconsistent. However, there are different levels of inconsistency, some of which are acceptable in solving real decision problems, some are not. Saaty [Saaty, 1980] defined the inconsistency ratio $C R$ as a positive linear transformation of $\lambda_{\text {max }}$. It is well known that $C R=0$ if and only if the matrix is consistent. According to Saaty, larger value of $C R$ indicates higher level of inconsistency and the 10 percent rule $(C R \leq 10 \%)$ separates acceptable matrices from unacceptable ones.

Incomplete pairwise comparison matrix was defined by Harker [Harker, 1987]. It is of the same form as (1)-(3) but one ore more elements, denoted here by *, are not given (or not given yet if we consider the filling in procedure):

$\mathbf{A}=$\begin{tabular}{|c|c|c|c|c|}
\hline 1 & $a_{12}$ & $*$ & $\ldots$ & $a_{1 n}$ \\
\hline $1 / a_{12}$ & 1 & $a_{23}$ & $\ldots$ & $*$ \\
\hline$*$ & $1 / a_{23}$ & 1 & $\ldots$ & $a_{3 n}$ \\
\hline$:$ & $:$ & $:$ & & $:$ \\
\hline $1 / a_{1 n}$ & $*$ & $1 / a_{3 n}$ & $\ldots$ & 1 \\
\hline
\end{tabular}

Variables $x_{1}, x_{2}, \ldots, x_{d}\left(x_{i}>0, i=1, \ldots, d\right)$ are introduced for the missing elements in the upper triangular part of $\mathbf{A}$. Their reciprocals, $1 / x_{1}, 1 / x_{2}, \ldots, 1 / x_{d}$ are written in the lower triangular part of $\mathbf{A}$. Let $\mathbf{x}$ denote the vector $\left(x_{1}, x_{2}, \ldots, x_{d}\right) . \mathbf{A}(\mathbf{x})$ is a complete pairwise comparison matrix for any values of $\mathbf{x}$. The total number of missing elements in matrix $\mathbf{A}$ is $2 d$.

$\mathbf{A}(\mathbf{x})=\mathbf{A}\left(x_{1}, x_{2}, \ldots, x_{d}\right)=$\begin{tabular}{|c|c|c|c|c|}
\hline 1 & $a_{12}$ & $x_{1}$ & $\ldots$ & $a_{1 n}$ \\
\hline $1 / a_{12}$ & 1 & $a_{23}$ & $\ldots$ & $x_{d}$ \\
\hline $1 / x_{1}$ & $1 / a_{23}$ & 1 & $\ldots$ & $a_{3 n}$ \\
\hline$:$ & $:$ & $:$ & & $:$ \\
\hline
\end{tabular}




\begin{tabular}{|l|l|l|l|l|}
\hline $1 / a_{1 n}$ & $1 / x_{d}$ & $1 / a_{3 n}$ & $\cdots$ & 1 \\
\hline
\end{tabular}

Based on the correspondence between the $C R$ inconsistency and $\lambda_{\max }$ of a pairwise comparison matrix, the generalization of the Eigenvector Method for the incomplete case is originated from the optimal solution of the eigenvalue minimization problem as follows [Shiraishi, Obata, Daigo, 1998; Shiraishi, Obata, 2002]:

$$
\min \left\{\lambda_{\max }(\mathbf{A}(\mathbf{x})) \mid \mathbf{x}>\mathbf{0}\right\}
$$

Note again that problem (5) is equivalent to the problem

$$
\min \{C R(\mathbf{A}(\mathbf{x})) \mid \mathbf{x}>\mathbf{0}\} .
$$

Inconsistency index $C M$ [Koczkodaj, 1993] of a $3 \times 3$ pairw ise comparison matrix [ [1, a, b] , [1/a, 1, c] , $[1 / \mathrm{b}, 1 / \mathrm{c}, 1]]$ is defined as $C M=\min \{|a-b / c| / a,|b-a c| / b,|c-b / a| / c\}=\min \{|1-b /(a c)|,|1-(a c) / b|\}$. In the case of matrices of higher orders, $C M(\mathbf{A})$ is defined as $\max \left\{C M\left(\mathbf{A}_{3 \times 3}\right) \mid \mathbf{A}_{3 \times 3}\right.$ is a $3 \times 3$ submatrix of $\mathbf{A}$ and $\mathbf{A}_{3 \times 3}$ itself is a pairwise comparison matrix $\}$.

$C M$ of an incomplete pairwise comparison matrix [Bozóki, Fülöp, Koczkodaj, 2011] is defined from the optimization problem as follows:

$$
\min \{C M(\mathbf{A}(\mathbf{x})) \mid \mathbf{x}>\mathbf{0}\} .
$$

Besides numerical inconsistency indices like $C R$ and $C M$, ordinal inconsistency has been also analyzed. Gass [Gass, 1998] investigated the property of ordinal transitivity $\left(a_{i j}>1\right.$ and $a_{j k}>1$ imply $\left.a_{i k}>1\right)$, which follows from cardinal trans itivity (4) but ordinal transitivity can also hold without cardinal transitivity. By Kéri's definition [Kéri, 2010], a pairwise comparison matrix $\mathbf{A}$ is qualitatively consistent if a consistent matrix $\mathbf{A}^{*}$ of the same size can be found so that $a_{i j}>1$ if and only if $a_{i j} *>1$ for any pairs of indices. A graph theoretic characterization of qualitatively consistent is also given in [Kéri, 2010].

\section{Methodology - incomplete matrices and graphs}

Assume that the decision maker compares the relative importance of $n$ criteria and we are able to observe the whole procedure of filling in the pairwise comparison matrix. In each comparison, a direct relation is defined between two criteria, namely, the estimated ratio of their weights. However, two criteria, not compared yet, consequently, having no direct relation, can be in indirect relation, through further criteria and direct relations. It is a natural idea to associate graph structures to (in)complete pairwise comparison matrices.

Given an (in)complete pairwise comparison matrix $\mathbf{A}$ of size $n \times n$, graph $G$ is defined as follows: $G:=(V, E)$, where $V=\{1,2, \ldots, n\}$, the vertices correspond to the objects to compare and $E=\left\{e(i, j) \mid a_{i j}\right.$ and $a_{j i}$ is given and $\left.i \neq j\right\}$, the undirected edges correspond to the matrix elements. There are no edges corresponding to the missing elements in the matrix. $G$ is an undirected graph. 
Example. Let a $6 \times 6$ pairwise comparison matrix be filled in by the decision maker, $\mathbf{B}_{5}, \mathbf{B}_{10}$ and $\mathbf{B}_{15}$ denotes the matrices having 5, 10 and 15 elements, respectively. Matrices $\mathbf{B}_{5}$ and $\mathbf{B}_{10}$ are incomplete and $\mathbf{B}_{15}$ is complete. Graphs associated are also drawn.

$\mathbf{B}_{5}=$\begin{tabular}{|c|c|c|c|c|c|}
\hline 1 & 2 & 5 & 7 & 9 & 9 \\
\hline $1 / 2$ & 1 & $*$ & $*$ & $*$ & $*$ \\
\hline $1 / 5$ & $*$ & 1 & $*$ & $*$ & $*$ \\
\hline $1 / 7$ & $*$ & $*$ & 1 & $*$ & $*$ \\
\hline $1 / 9$ & $*$ & $*$ & $*$ & 1 & $*$ \\
\hline $1 / 9$ & $*$ & $*$ & $*$ & $*$ & 1 \\
\hline
\end{tabular}

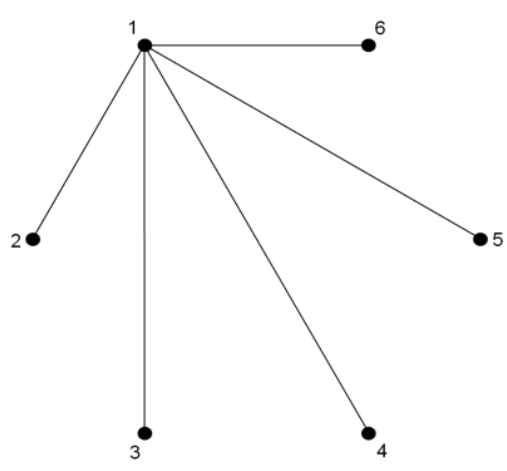

$\mathbf{B}_{10}=$\begin{tabular}{|c|c|c|c|c|c|}
\hline 1 & 2 & 5 & 7 & 9 & 9 \\
\hline $1 / 2$ & 1 & 3 & 6 & 8 & 9 \\
\hline $1 / 5$ & $1 / 3$ & 1 & 4 & $*$ & $*$ \\
\hline $1 / 7$ & $1 / 6$ & $1 / 4$ & 1 & $*$ & $*$ \\
\hline $1 / 9$ & $1 / 8$ & $*$ & $*$ & 1 & $*$ \\
\hline $1 / 9$ & $1 / 9$ & $*$ & $*$ & $*$ & 1 \\
\hline
\end{tabular}

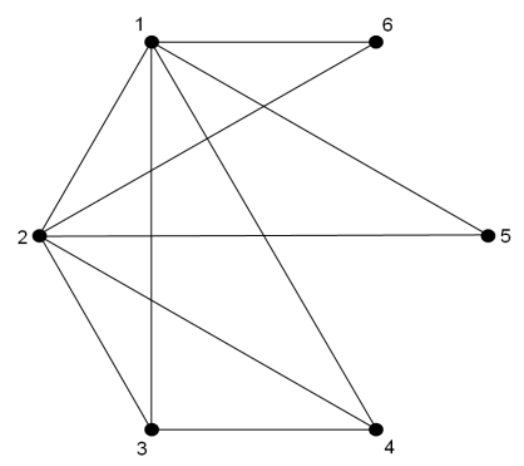

$\mathbf{B}_{15}=$\begin{tabular}{|c|c|c|c|c|c|}
\hline 1 & 2 & 5 & 7 & 9 & 9 \\
\hline $1 / 2$ & 1 & 3 & 6 & 8 & 9 \\
\hline $1 / 5$ & $1 / 3$ & 1 & 4 & 6 & 7 \\
\hline $1 / 7$ & $1 / 6$ & $1 / 4$ & 1 & 3 & 5 \\
\hline $1 / 9$ & $1 / 8$ & $1 / 6$ & $1 / 3$ & 1 & 2 \\
\hline $1 / 9$ & $1 / 9$ & $1 / 7$ & $1 / 5$ & $1 / 2$ & 1 \\
\hline
\end{tabular}

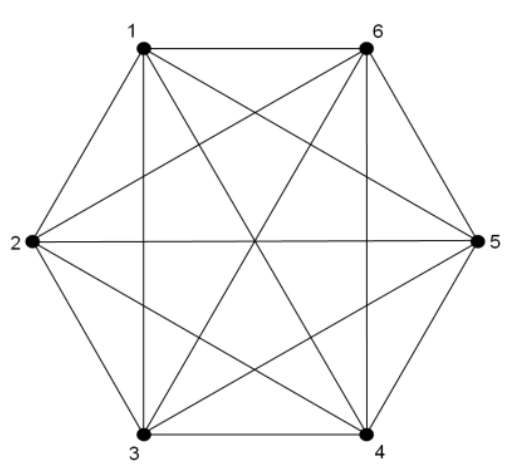




\section{Summary of the results}

Theoretical results are related to the connectedness of the graph associated to the incomplete pairwise comparison matrix. Connectedness is a natural necessary condition, otherwise the problems breaks into several parts the weights of which can change independently from each other.

The orem [Bozóki, Fülöp, Rónyai, 2010]: The optimal solution of the problem (6) is unique if and only if the graph corresponding to the incomplete pairwise comparison matrix is connected.

It is also shown [Bozóki, Fülöp, Koczkodaj, 2011] that problem (7) can be reduced to an LP problem the solution of which is found fast.

By solving problems (6) and/or (7) real time, the decision maker may get automatic lower tight bounds of $C R / C M$ inconsistency levels in each step of the filling in process. These kinds of information are useful for an immediate recognition of possible misprints. Warning rules and thresholds are under construction as they can be determined based on empirical pairwise comparison matrices.

Pairwise comparison matrices that can be made consistent by the modification of a few $(1,2,3)$ elements are characterized in [Bozóki, Fülöp, Poesz, 2010]. Empirical examples collected from case studies show that these kinds of matrices are not only of theoretical interest but they are present in practice as well [Poesz, 2009].

Algorithms for giving feedback on ordinal (in)consistency are of the same importance as on $C R / C M$ inconsistencies. The extensions of the theorems and propositions cited from Gass [Gass, 1998] and Kéri [Kéri, 2010] to the incomplete case are of the present and future research.

\section{Conclusion}

There are several types of inconsistency, a number of indices have been defined for both ordinal and cardinal violations of the well known equation $a_{i j} a_{j k}=a_{i k}$ (for all indices $i, j, k=1, \ldots, n$ ). We focused on two of the cardinal indices, $C R$ and $C M$, both are extended to the incomplete case. The decision maker gets a lower bounds for the $C R / C M$-inconsistency level in each step when filling in the pairwise comparison matrix, that can be used for detecting misprints in real time.

\section{REFERENCES}

Bozóki, S., Dezső, L., Poesz, A., \& Temesi, J. (2011). Pairwise comparison matrices: an empirical research, Proceedings of The International Symposium on the Analytic Hierarchy Process (ISAHP), 15-18 June, 2011, Sorrento (Naples), Italy.

Bozóki, S., Fülöp, J., \& Koczkodaj, W.W. (2011). LP-based inconsistency analysis of incomplete pairwise comparis on matrices (submitted).

Bozóki, S., Fülöp, J., \& Poesz, A. (2010): On pairwise comparison matrices that can be made consistent by the modification of a few elements, Central European Journal of Operations Research, in press, published online: 24 February 2010, DOI 10.1007/s10100-010-0136-9. 
Bozóki, S., Fülöp, J., \& Rónyai, L. (2010). On optimal completions of incomplete pairwise comparison matrices. Mathematical and Computer Modelling, 52, 318-333.

Gass, S.I. (1998). Tournaments, transitivity and pairwise comparison. Journal of the Operational Research Society 49, 616-624.

Harker, P.T. (1987). Incomplete pairwise comparisons in the analytic hierarchy process. Mathematical Modelling, 9(11), 837-848.

Kéri, G. (2010). On qualitatively consistent, transitive and contradictory judgment matrices emerging from multiattribute decision procedures, Central European Journal of Operations Research, in press, published online: 24 February 2010, DOI 10.1007/s10100-010-0138-7.

Poesz, A. (2009). Empirical pairwise comparison matrices (EPCM) - an on-line collection from real decisions, http://www.uni-corvinus.hu/index.php?id=29191; http://www.sztaki.hu/ bozoki/epcm

Saaty, T.L. (1980). The analytic hierarchy process, McGraw Hill, New York.

Shiraishi, S., Obata, T., \& Daigo, M. (1998). Properties of a positive reciprocal matrix and their application to AHP. Journal of the Operations Research Society of Japan, 41(3), 404-414.

Shiraishi, S., \& Obata, T. (2002). On a maximization problem arising from a positive reciprocal matrix in AHP. Bulletin of Informatics and Cybernetics, 34(2), 91-96. 